\title{
Dihydroartemisinin and its anticancer activity against endometrial carcinoma and cervical cancer: involvement of apoptosis, autophagy and transferrin receptor
}

\author{
Tian $\underline{\text { Tang }}^{1}$, MD, Qingjie ${\underline{X i a^{2}}}^{2}$ MD, Mingrong $\underline{X i}^{1}$, MD
}

INTRODUCTION Dihydroartemisinin (DHA) is a first-line antimalarial drug with relatively low toxicity. DHA has been speculated to possess a broad-spectrum antitumour effect. However, the potential value of DHA for the treatment of endometrial carcinoma or cervical cancer is unclear.

METHODS We used human endometrial cancer cells and cervical cancer cells to assess whether DHA alone or when combined with cisplatin would induce cell death. We aimed to elucidate the role of autophagy in DHA-induced cytotoxicity in both endometrial and cervical cancer cells, and explore the impact of DHA treatment on cell proliferation, apoptosis and autophagy. RESULTS DHA alone or in combination with cisplatin induced cell death in a dose- and time-dependent manner. Caspase-3 mRNA and cleaved caspase-3 protein levels were markedly elevated following DHA treatment either in the presence or absence of cisplatin, suggesting a role of apoptosis in DHA-induced cell death. DHA treatment activated the autophagic pathway, as evidenced by increased monodansylcadaverine-positive staining, elevated microtubule-associated protein 1 light chain 3 (LC3)-II/LC3-I ratio, and enhanced p62/sequestosome 1 degradation. Inhibition of autophagy by 3-methyladenine further enhanced the cytotoxicity of DHA towards tumour cells. mRNA levels of transferrin receptor (TfR) were suppressed upon DHA treatment and knockdown of TfR by RNA interference caused further DHA induction of cancer cell death.

CONCLUSION Our results suggest a clinical value for DHA in the treatment of endometrial carcinoma and cervical cancer. Our data revealed possible anticancer mechanisms of DHA that involve regulating apoptosis, autophagy pathway and levels of TfR.

Keywords: apoptosis, autophagy, cervical cancer, dihydroartemisinin (DHA), endometrial carcinoma

\section{INTRODUCTION}

Endometrial carcinoma and cervical cancers are the third and fourth most common causes of cancer-related death in women worldwide, respectively. ${ }^{(1,2)}$ Because of the widespread use of cervical screening programmes in developed countries, the incidence of cervical cancer has been reduced. ${ }^{(1)}$ However, in the past decades, rates of endometrial cancer have risen in a number of countries, likely due to the increasingly ageing populations and growing obesity rates. ${ }^{(2,3)}$ Even with current advanced treatment strategies, the side effects and drug resistance of chemotherapeutic agents have limited the efficacy of treatment. ${ }^{(3-5)}$ It is therefore of significant importance to discover novel agents with less severe side effects and lower drug resistance.

Autophagy is a lysosomal pathway involved in the degradation of cytoplasmic macromolecules and organelles. ${ }^{(6,7)}$ It plays a dual role in cancer development and treatment. Increased autophagy promotes the generation of metabolic substrates, which maintains cancer homeostasis, and thereby limits chemotherapy cytotoxicity. However, excessive self-digestion of essential cellular components can be toxic to cancer cells. The microtubule-associated protein 1 light chain 3 (LC3) system is important for the transport and maturation of the autophagosome.
Accordingly, this study aimed to elucidate the role of autophagy in dihydroartemisinin (DHA)-induced cytotoxicity in both cervical and endometrial cancer cells.

Transferrin receptor (TfR) is a serum glycoprotein that transfers iron, a co-factor for DNA synthesis, from the extracellular matrix to the intracellular space. ${ }^{(8,9)}$ Because of rapid proliferation, the demand for iron in cancer cells is much greater than in normal cells, resulting in elevated TfR expression in cancer cells. ${ }^{(8,9)}$ Apoptosis can be classified into extrinsic and intrinsic pathways. However, the potential correlation between TfR and the apoptotic pathway is unknown. Additionally, the relationship between key factors in apoptosis, including caspase-3, and TfR is still unclear.

$\mathrm{DHA}$, an artemisinin derivative, was originally an effective agent against malaria, with minimal side effects. Later, DHA was reported to have anticancer effects. ${ }^{(10)}$ It has been found that DHA displays antiproliferative effects against various tumour cell lines, including cancers of the lung, breast, colon, pancreas, liver, ovary and prostate, as well as some anticancer drug-resistant cell lines, ${ }^{(8,11)}$ and that it inhibits cell growth while inducing apoptosis in a dose- and time-dependent manner. ${ }^{(12)}$ However, some limitations constrain its clinical application, such as low bioavailability caused by its poor solubility. ${ }^{(13)}$ The antitumour

${ }^{1}$ Department of Obstetrics and Gynecology, West China Second Hospital, Key Laboratory of Birth Defects and Related Diseases of Women and Children (Sichuan University), Ministry of Education, ${ }^{2}$ West China Laboratory of Molecular Genetics, Sichuan University, Chengdu, China

Correspondence: Dr Mingrong Xi, Chief, Department of Gynecology and Obstetrics, West China Second University Hospital, Key Laboratory of Birth Defects and Related Diseases of Women and Children, Ministry of Education, Chengdu, China. qmrjzzjzz@126.com 
mechanisms of DHA are still not fully understood. Inhibition of tumour cell growth by DHA may partially be due to the production of reactive oxygen species. ${ }^{(14,15)}$ DHA may also affect expression and function of adhesion proteins, matrix metalloproteinases and tissue inhibitors to inhibit cancer metastasis. However, the role of DHA in autophagy and the mechanism by which DHA induces autophagy, apoptosis and its association with TfR remain unclear.

Cisplatin is currently the best known chemotherapeutic drug available for the treatment of ovarian cancer. However, for cisplatin-resistant cancer cells, new effective anticancer drugs are urgently needed.

LC3 is a soluble protein that is distributed ubiquitously in mammalian tissues and cultured cells. Upon autophagic stimulation, a cytosolic form of LC3 (LC3-I) is conjugated to phosphatidylethanolamine to form LC3-phosphatidylethanolamine conjugate (LC3-II), which is recruited to isolation membranes to facilitate the generation of autophagosomes. Autophagosomes then fuse with lysosomes to form autolysosomes, and intraautophagosomal components are degraded by lysosomal hydrolases. p62 plays a key role in the formation of cytoplasmic proteinaceous inclusion. ${ }^{(16)}$ The p62 protein recognises toxic cellular waste, which is then scavenged by a sequestration process known as self-eating or autophagy. The p62 protein itself is also a substrate of autophagy. Increased autophagic activity results in rapid degradation of p62, whereas a lack of autophagy leads to accumulation of p62. ${ }^{(17)}$

In the present study, we explored the impact of DHA treatment on cell proliferation, apoptosis and autophagy in endometrial and cervical cancer cells. Our results would provide further evidence to support DHA as a possible anticancer drug for the treatment of endometrial carcinoma and cervical cancer.

\section{METHODS}

Ishikawa cells (Shanghai Bogoo Biotechnology Co, Shanghai, China), which are human endometrial adenocarcinoma cell lines, were cultured in complete Dulbecco's modified Eagle's medium (DMEM) with 5\% fetal bovine serum (FBS), $100 \mathrm{U} / \mathrm{mL}$ penicillin and $100 \mathrm{U} / \mathrm{mL}$ streptomycin at $37^{\circ} \mathrm{C}$ in a humidified incubator, with an atmosphere of $5 \%$ carbon dioxide $\left(\mathrm{CO}_{2}\right)$ in compressed air. HeLa cells - human cervical cancer cell lines - obtained from the American Type Culture Collection (ATCC, Manassas, VA, USA) were grown in DMEM supplemented with $10 \%$ FBS.

Total RNA was isolated using the TRIzol ${ }^{\mathrm{TM}}$ reagent (Invitrogen, San Francisco, California, USA) and reverse transcribed with the RevertAid $^{\mathrm{TM}}$ First Strand CDNA Synthesis Kit (MBI Fermentas Inc, Pickering, ON, Canada). Quantitative reverse transcriptionpolymerase chain reaction (RT-PCR) was performed using the FTC-2000 real-time PCR machine (FTC-2000; Funglyn Biotech Inc, Markham, Ontario, Canada). All mRNA data was normalised to $\beta$-actin mRNA levels. A dissociation curve was obtained to verify the specificity of the amplification. Primer sequences used for PCR are shown in Box 1.

Results were normalised internally using the $\mathrm{Ct}$ number of the housekeeping gene $A C T B: \Delta \mathrm{Ct}$ (sample) $=(\mathrm{Ct}$ sample $)-$ (Ct $A C T B)$.
Box 1. Primer sequences for polymerase chain reaction:

TfR F: 5'-CAGCATTCTCTAACTTGTTTGG-3',

TfR R: 5'-GTTTCATCTCCACATGACTGTT-3',

TfR TM: 5'-ACCCGGTTCAGCCTGGCTCGGC-3' (99 bp);

ACTB F: 5'-GAAGATCAAGATCATTGCTCCT-3',

ACTB R: 5'-TACTCCTGCTTGCTGATCCACA-3',

ACTB TM: 5'-TCACTGTCCACCTTCCAGCAGA-3' (111 bp);

Caspase-3 F: 5'-TTCAGGCCTGCCGTGGTACA-3',

Caspase-3 R: 5'-CCAAGAATAATAACCAGGTGCT-3' and

Caspase-3 TM: 5'-CACCACTGTCTGTCTCAATGCC-3' (140 bp)

The mean Ct of TfR RNA from the blank was set to a relative quantity (RQ) value of 1 using the comparative Ct method ( $\Delta \Delta \mathrm{Ct}$ ), which was calculated as: $\Delta \Delta \mathrm{Ct}$ (sample) $=\Delta \mathrm{Ct}$ (sample) $-\Delta \mathrm{Ct}$ (blank), and $\mathrm{RQ}=2-\Delta \Delta \mathrm{Ct}$.

Cell viability was determined through the MTT (3-[4,5-dimethyl-2-thiazolyl]-2,5-diphenyl-2-H-tetrazolium bromide) assay (Amresco, Solon, Ohio, USA). Ishikawa cells $\left(5 \times 10^{4}\right.$ cell $\left.\mathrm{s} / \mathrm{mL}\right)$ were incubated with various concentrations of DHA and/or cisplatin in complete medium at $37^{\circ} \mathrm{C}$ under $5 \% \mathrm{CO}_{2}$. MTT solution $(5 \mathrm{mg} / \mathrm{mL}$ in phosphate-buffered saline [PBS]) was then added to the medium and the resultant formazan crystals were dissolved in dimethyl sulfoxide. Absorbance at $570 \mathrm{~nm}$ was recorded using a SpectraMax 190 microplate reader (SpectraMax 190 Microplate Reader; Molecular Devices, San Jose, CA, USA). The cell growth inhibitory ratio was calculated using the following formula and described with a cell growth inhibitory curve: cell growth inhibitory ratio $=[1-$ (average absorbance of experimental group/average absorbance of control group) $] \times 100 \%$. Each experiment was performed three times and the half-maximal (50\%) inhibitory concentration was calculated based on the curve.

Autophagic structures were visualised by monodansylcadaverine (MDC) staining. In brief, Ishikawa cells $\left(5 \times 10^{4} \mathrm{cell} \mathrm{s} / \mathrm{mL}\right)$ plated onto six-well culture plates were stained with $0.05 \mathrm{mM}$ MDC (Sigma, St. Louis, MO, USA). MDC-stained cells were visualised with a confocal microscope (Fluoview FV10i; Olympus, Center Valley, PA, USA).

Ishikawa cells grown to $50 \%$ confluence were transfected with control or TfR siRNA (small interfering RNA; RiboBio, Guangzhou, Guangdong, China) using Lipofectamine 2000 (Life Technologies, Grand Island, NY, USA) as per the manufacturer's instruction. Following six hours of incubation, the medium was replaced with DMEM containing $10 \%$ FBS. After 48 hours at $37^{\circ} \mathrm{C}$ and $5 \% \mathrm{CO}_{2}$, cells were used in the subsequent experiments.

For Western blot analysis, cells were treated with various concentrations of DHA, as indicated. Cells were washed with ice-cold PBS and then lysed in Modified Oncogene Science lysis buffer (50 mM sodium pyrophosphate, $50 \mathrm{mM}$ sodium fluoride, $50 \mathrm{mM}$ sodium chloride, $5 \mathrm{mM}$ EDTA [ethylenediaminetetraacetic acid], $5 \mathrm{mM}$ EGTA [ethylene glycol-bis $\{\beta$-aminoethyl ether $\}$ $\mathrm{N}, \mathrm{N}, \mathrm{N}^{\prime}, \mathrm{N}^{\prime}$-tetraacetic acid, or egtazic acid], $100 \mu \mathrm{M}$ sodium orthovanadate $\left[\mathrm{Na}_{3} \mathrm{VO}_{4}\right], 10 \mathrm{mM}$ HEPES [4-\{2-hydroxyethyl\}-1piperazineethanesulfonic acid] and $0.1 \%$ Triton X-100). Protein concentration was measured using the Bradford assay. Equivalent amounts of total protein were resolved using 15\% sodium dodecyl 
sulphate-polyacrylamide gel electrophoresis and then transferred to nitrocellulose membranes (Millipore Co, Billerica, MA, USA). The membranes were blocked in 5\% non-fat milk in Tris-buffered saline plus Tween 20 buffer (10 mM Tris pH 7.5, 150 mM sodium chloride, $0.1 \%$ Tween 20 ) for one hour at room temperature, followed by one-hour incubation with a primary antibody and a subsequent one-hour incubation with horseradish peroxidase-conjugated secondary antibody (Sigma, St Louis, MO, USA). Immunoreactive bands were detected using enhanced chemiluminescence (Pierce Biotechnology, Rockford, IL, USA). Primary antibodies used in this study included anti-caspase-3 (Santa Cruz Biotechnology, Santa Cruz, CA, USA), anti-p62 (PROGEN Biotechnik GmbH, Maaßstraße, Heidelberg, Germany) and anti-microtubule-associated protein LC3 (MBL International, Woburn, MA, USA).

The appearance of phosphatidylserine on the extracellular side of the cell membrane was quantified using annexin $\mathrm{V} /$ propidium iodide (PI) staining. After $20 \mu \mathrm{M}$ DHA treatment for 12 hours or 24 hours, control cells and DHA-treated cells were stained with $5 \mu \mathrm{L}$ of annexin V-fluorescein isothiocyanate and $10 \mu \mathrm{L} \mathrm{PI}(10 \mu \mathrm{g} / \mathrm{mL})$ for ten minutes at room temperature, as recommended by the manufacturer (Thermofisher, San Francisco, CA, USA). Cells were subjected to fluorescence-activated cell sorting analysis using a flow cytometer (BD FACSymphony, San Jose, CA, USA) with apoptotic cells being annexin $\mathrm{V}$-positive/PI-negative.

For detection of cleaved caspase-3 protein levels by enzymelinked immunosorbent assay (ELISA), the human/mouse cleaved caspase-3 (Asp175)-DuoSet ELISA kit (R\&D Systems, Abingdon, England, UK) was used in accordance with the manufacturer's instruction.

Data was expressed as mean \pm standard deviation of at least three independent experiments. Data was analysed using Student's $t$-test when only two groups were present or assessed using one-way analysis of variance when more than two groups were compared. Differences between groups were considered to be statistically significant at $p<0.05$.

\section{RESULTS}

Anticancer activity was seen in human endometrial adenocarcinoma cells when they were treated with various concentrations of DHA or cisplatin, either alone or in combination, over a period of 96 hours (Fig. 1). Cell survival, as measured by MTT assay, was inhibited by DHA in a dose- and time-dependent manner. The half-maximal inhibitory concentrations, when extrapolated from the dose-response curve of Ishikawa cells for DHA alone, were $13.6 \mu \mathrm{M}, 30.5 \mu \mathrm{M}$ and $112.7 \mu \mathrm{M}$, respectively, at 24 hours, 48 hours and 96 hours.

Results of the MTT assay indicated that treatment of Ishikawa cells with DHA at $20 \mu \mathrm{M}, 40 \mu \mathrm{M}$ or $80 \mu \mathrm{M}$ for 96 hours exhibited a potent antiproliferative effect ( $p<0.01$; Fig. 1a). It also revealed that DHA at $40 \mu \mathrm{M}$ and $80 \mu \mathrm{M}$ inhibited cancer cells for 48 hours and 24 hours, respectively ( $<<0.05$; Fig. 1a). Treatment with cisplatin at $2 \mu \mathrm{M}, 20 \mu \mathrm{M}$ or $200 \mu \mathrm{M}$ for 96 hours exhibited a potent antiproliferative effect ( $p<0.01$; Fig. 1b). However, no such inhibitory action was seen in cells treated with DHA and cisplatin in combination (Fig. 1C).
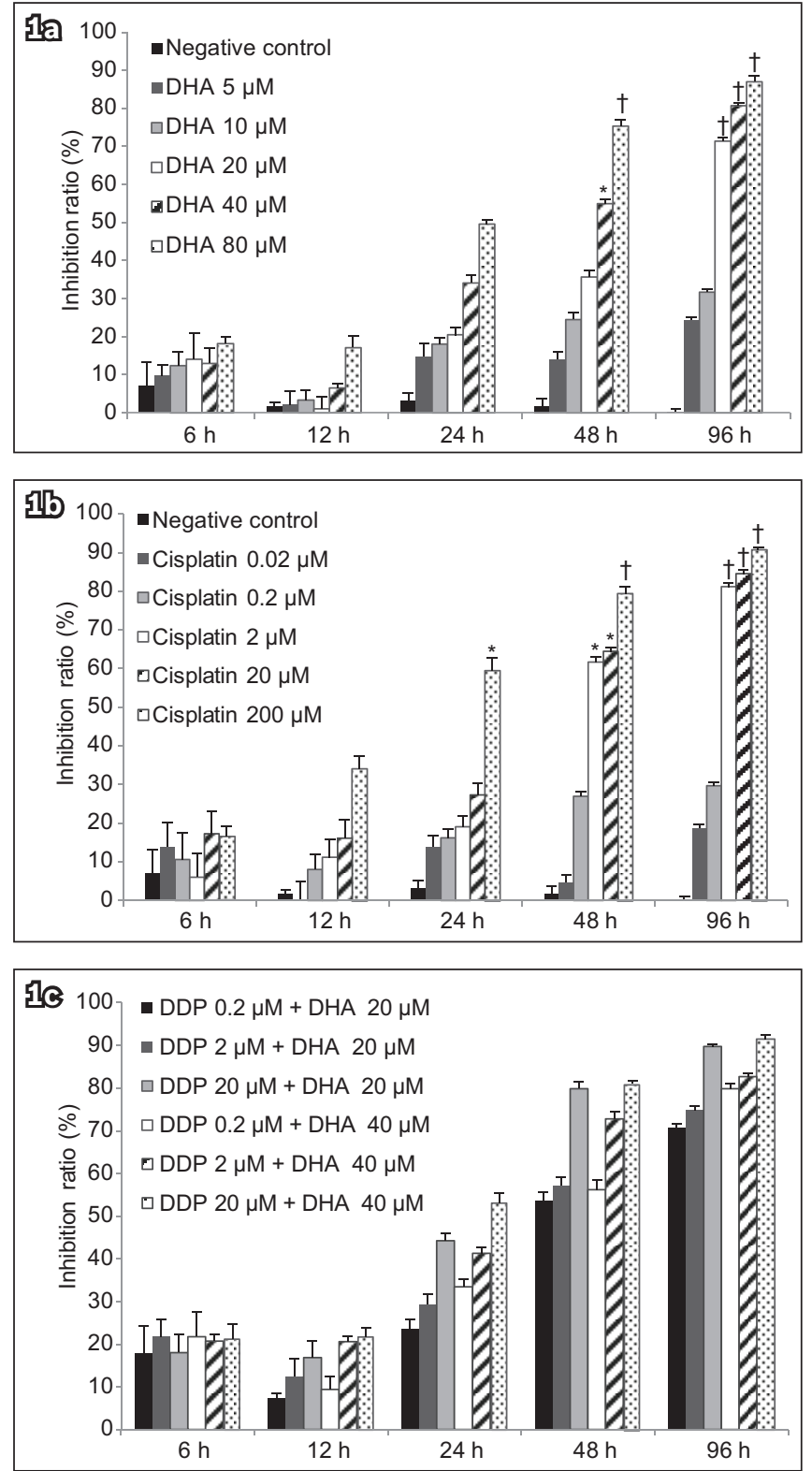

Fig. 1 Charts show cell viability following treatment of Ishikawa cells with varying concentrations of (a) DHA alone, (b) cisplatin alone, or (c) DHA and cisplatin determined by the MTT assay. Inhibitory effect of DHA on cell survival was expressed as a percentage of the inhibition ratio. Data was expressed as mean \pm standard deviation of three independent experiments. ${ }^{*} p<0.05$ was statistically significant when compared to control cells. $t p<0.01$ was statistically significant when compared to control cells. DHA: dihydroartemisinin; MTT: 3-(4,5-dimethyl-2-thiazolyl)-2,5-diphenyl2-H-tetrazolium bromide assay

The quantitative results of flow cytometry (Fig. 2), which was performed to investigate whether reduced cell survival upon treatment with DHA and/or cisplatin of Ishikawa cells was associated with increased apoptosis and necrosis, showed that the addition of DHA, or a combination of DHA and cisplatin, led to a significant increase in the number of cell apoptosis and/or necrosis at 24 hours after treatment $(p<0.05)$. This indicated that treatment with DHA induced increased apoptosis and necrosis.

The mRNA levels of caspase- 3 and TfR in human endometrial adenocarcinoma cells treated with DHA alone, cisplatin alone or DHA in combination with cisplatin were measured using RT$\mathrm{PCR}$, with $A C T B$ as a reference gene. We found that TfR mRNA 

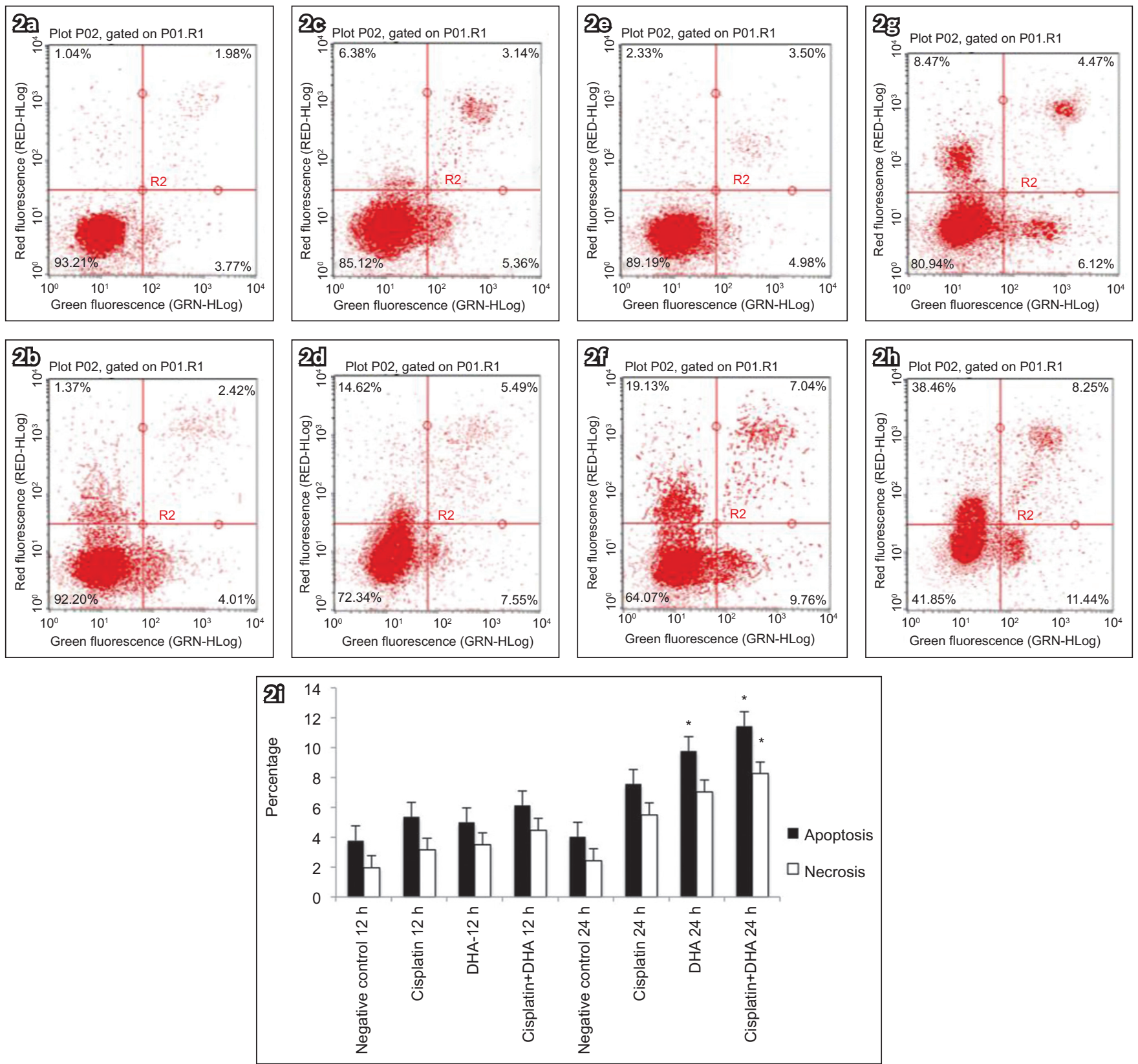

Fig. 2 (a-h) Flow cytometry charts show that DHA triggered apoptosis in human endometrial cancer cells. Ishikawa cells were treated with (a) vehicle for 12 hours, (b) vehicle for 24 hours, (c) $20 \mu \mathrm{M}$ cisplatin for 12 hours, (d) $20 \mu \mathrm{M}$ cisplatin for 24 hours, (e) $40 \mu \mathrm{M}$ DHA for 12 hours, (f) $40 \mu \mathrm{M}$ DHA for 24 hours, (g) $40 \mu \mathrm{M}$ DHA and $20 \mu \mathrm{M}$ cisplatin for 12 hours, or (h) $40 \mu \mathrm{M}$ DHA and $20 \mu \mathrm{M}$ cisplatin for 24 hours. (i) Chart shows significantly more cell apoptosis and/or necrosis after treatment (bars indicate mean \pm standard deviation from three independent experiments). ${ }^{*} p<0.05$ was statistically significant when compared to control cells. DHA: dihydroartemisinin

expression was decreased in groups with DHA at 36 hours ( $\mathrm{p}<0.05$; Fig. 3a). Caspase-3 mRNA expression in cells treated with DHA at 36 hours was significantly increased when compared to controls ( $p<0.05$; Fig. 3a). Results of RT-PCR suggested that DHA induced apoptosis more potently than cisplatin, possibly with the assistance of TfR.

To further determine whether DHA triggered caspase activation in Ishikawa cells, protein levels of cleaved caspase-3 were examined using ELISA. As shown in Fig. 3b, the addition of cisplatin, DHA or both led to significantly higher levels of cleaved caspase-3 when compared with vehicle-treated control cells ( $p$ $<0.05$ ). Levels of cleaved caspase- 3 were markedly higher at 24 hours or 12 hours of treatment when compared with the negative control $(p<0.05)$. No significant difference was found between cells treated with DHA and cisplatin. However, the highest levels of cleaved caspase-3 for any time point were observed in cells treated with both DHA and cisplatin $(\mathrm{p}<0.05)$.

Ishikawa cells treated with $40 \mu \mathrm{M} \mathrm{DHA}$ alone, $20 \mu \mathrm{M}$ cisplatin alone, or $40 \mu \mathrm{M} \mathrm{DHA}$ and $20 \mu \mathrm{M}$ cisplatin for 12 hours were stained with MDC to determine a possible association between DHA and autophagy, and the numbers of MDC-positive dots in cells counted (Fig. 4). MDC-positive staining was comparable among cells treated with DHA alone and DHA and cisplatin, and this significantly increased when compared with cells treated with cisplatin and control cells $(p<0.01)$. However, the number of MDC-positive dots was not significantly increased in cells 

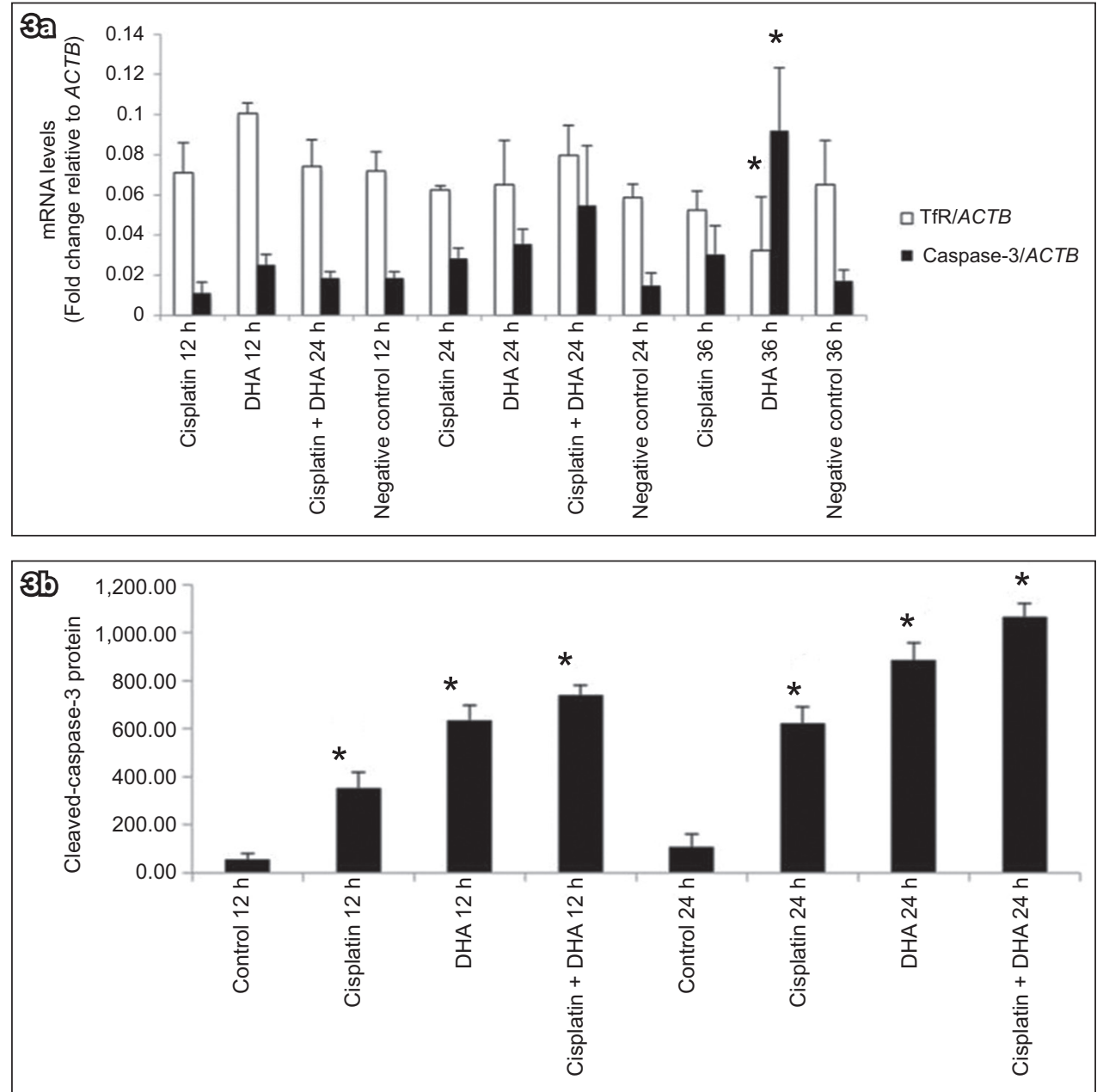

Fig. 3 (a) Chart shows mRNA levels of caspase- 3 and TfR in Ishikawa cells treated with $40 \mu \mathrm{M}$ DHA or $20 \mu \mathrm{M}$ cisplatin alone or in combination, as assessed by qRT-PCR (normalised to the housekeeping gene ACTB). DHA treatment induced increased cleavage of caspase-3 in human endometrial cancer cells. (b) Chart shows levels of cleaved caspase-3 protein, which were measured by ELISA to determine whether DHA treatment triggered caspase activation in Ishikawa cells. Addition of DHA and/or cisplatin led to significantly higher cleaved caspase-3 levels. Bars represent mean \pm standard deviation from three independent experiments. ${ }^{*} p<0.05$ was statistically significant when compared to control cells. DHA: dihydroartemisinin; ELISA: enzyme-linked immunosorbent assay; qRT-PCR: real-time quantitative reverse transcription polymerase chain reaction; TfR: transferrin receptor

treated with cisplatin when compared to controls. Labelling of autophagic cells with MDC showed that DHA greatly induced autophagy.

The effects of autophagy inhibition and gene silencing of TfR on DHA-induced cell death were further examined using MTT assay. Ishikawa cells were treated with autophagy inhibitor 3-methyladenine (3-MA; $5 \mathrm{mM}$ ) or transfected with $10 \mu \mathrm{M}$ TfRsiRNA in the presence or absence of $40 \mu \mathrm{M}$ DHA (Fig. 5). The addition of 3-MA induced cytotoxicity after DHA treatment for 48 hours $(p<0.05)$, suggesting a prosurvival role for autophagy during DHA treatment. It was also observed that gene silencing of TfR further reduced the survival of cells after 48 hours of DHA treatment $(p<0.05)$, indicating that knockdown of TfR promoted DHA-induced cell death.

Finally, we examined the effects of DHA on apoptosis and autophagy in HeLa cells. As shown in Fig. 6a, similar to the observations in human endometrial cancer cells, treatment with various concentrations of DHA for 48 hours resulted in a dose-dependent reduction of cell survival. Addition of DHA also caused the cleavage of caspase-3, suggesting an activation of the apoptosis pathway (Fig. 6b). Moreover, the effects of DHA on the autophagy pathway in HeLa cells, when examined by Western blotting, demonstrated that the addition of DHA led to increased conversion of LC3-I to LC3-II (Fig. 6c) and accelerated degradation of p62 (Fig. 6d), which is a substrate of autophagy. Together, our results indicated that DHA triggered cell death in human cervical cancer cells by promoting the apoptosis pathway and that DHA treatment also induced autophagy pathways.

\section{DISCUSSION}

TfR may be upregulated in order to mediate endocytosis of transferrin loaded with increased concentrations of iron. ${ }^{(17,18)} \mathrm{We}$ found that DHA decreased TfR mRNA expression and that this knockdown of TfR further enhanced the cytotoxic activity of DHA. Most cancer cells have greater numbers of iron-attracting TfR on their cell surface when compared to normal cells. ${ }^{(18)}$ Thus, we speculated that knockdown of TfR leads to the death of Ishikawa cells due to lack of iron uptake. DHA could possibly interact with the upregulated iron receptor TfR to form free radicals, thereby acting as an effective anticancer drug. ${ }^{(8)}$ Our results indicated that knockdown of TfR may synergistically enhance the anticancer 

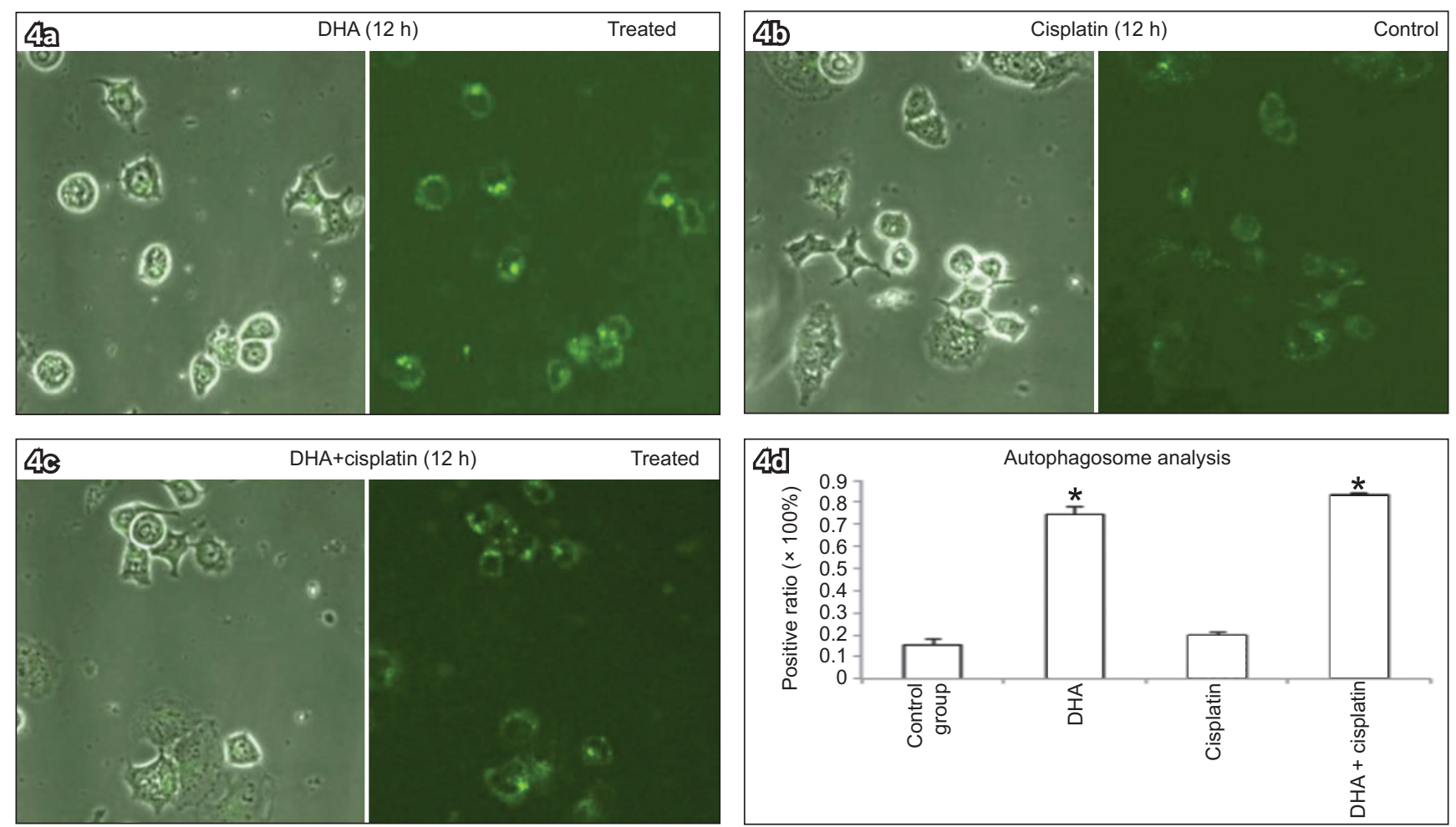

Fig. 4 Monodansylcadaverine (MDC) staining of Ishikawa cells treated with (a) $40 \mu \mathrm{M} \mathrm{DHA}$ alone, (b) $20 \mu \mathrm{M}$ cisplatin alone, or (c) both $40 \mu \mathrm{M}$ DHA and $20 \mu \mathrm{M}$ cisplatin for 12 hours to determine association between DHA and autophagy show elevated numbers of MDC-positive autophagosomes, with DHA inducing increased positive MDC staining in cells (MDC staining, $\times 400$ ). (d) Chart shows number of MDC-positive dots scored in 100 cells (bars represent mean \pm standard deviation from three independent measurements). ${ }^{*} p<0.01$ was statistically significant when compared to control cells. DHA: dihydroartemisinin

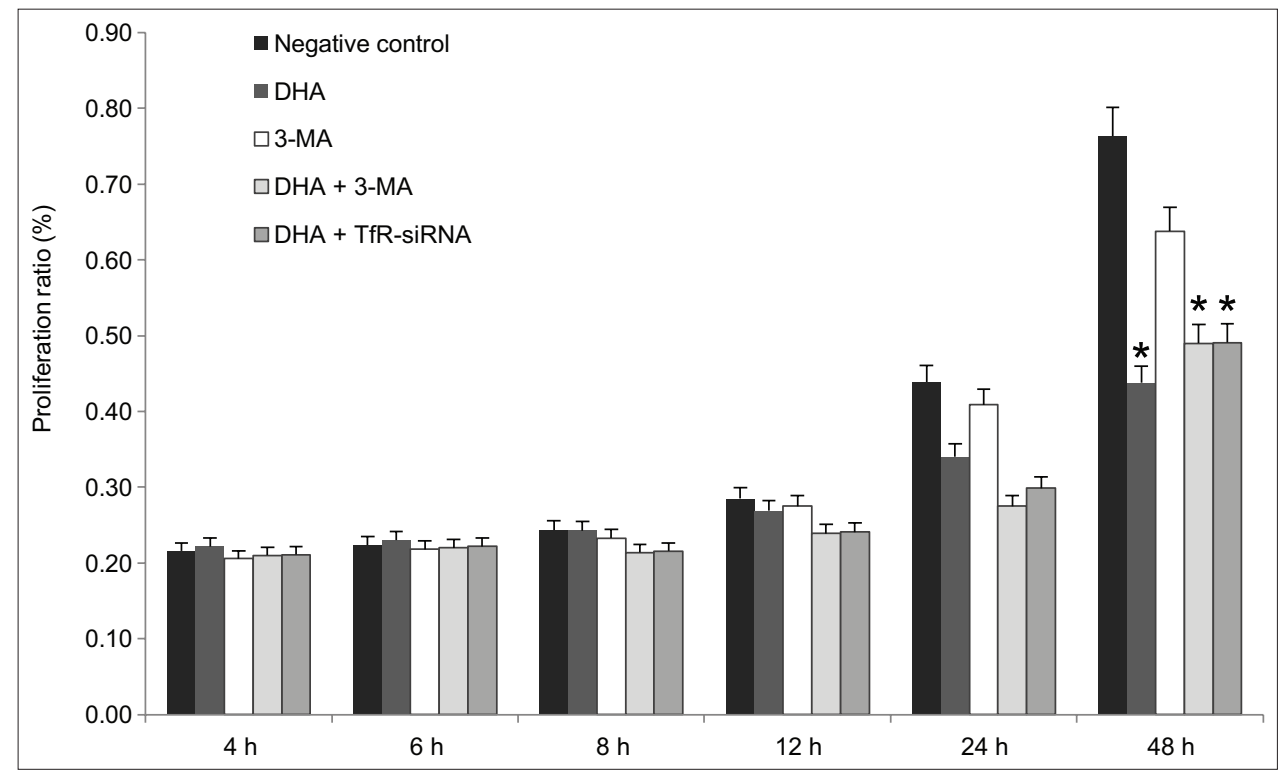

Fig. 5 Chart about the effects of autophagy inhibition and gene silencing of TfR on DHA-induced cell death, when examined using MTT assay, showed that knockdown of TfR or inhibition of autophagy enhanced cell death due to DHA. Inhibitory effect of DHA on cell survival was expressed as percentage of inhibition ratio; bars represent mean \pm standard deviation from three independent experiments. * $p<0.05$ was statistically significant when compared to control cells. 3-MA: 3-methyladenine; DHA: dihydroartemisinin; MTT: 3-(4,5-dimethyl-2-thiazolyl)-2,5-diphenyl-2-H-tetrazolium bromide assay; SiRNA: small interfering RNA; TfR: transferrin receptor

effects of DHA through depletion of iron metabolism in cervical and endometrial carcinoma cells.

DHA was also reported to cause an arrest of cell cycle progression. ${ }^{(19)}$ It was previously shown that DHA treatment resulted in a gradual $\mathrm{G} 1$ and G2/M arrest with increased doses of both $\mathrm{G} 1$ and $\mathrm{G} 2$ peaks, while DHA decreased G0/G1 phase doses in ovarian cell lines (OVCA432 and SKOV3 cells). ${ }^{(20)}$ Meanwhile, cell cycle analysis showed that DHA inhibited cell proliferation by inducing cell cycle arrest at the $S$ phase in a concentrationdependent manner (SKOV3/DDP cells). ${ }^{(16)}$ Further studies on cycle analysis are warranted for the many cell cycle genes and proteins, including CDKN1A, CCNE1, CDKN1C, CDKN2D and Cdk1. 

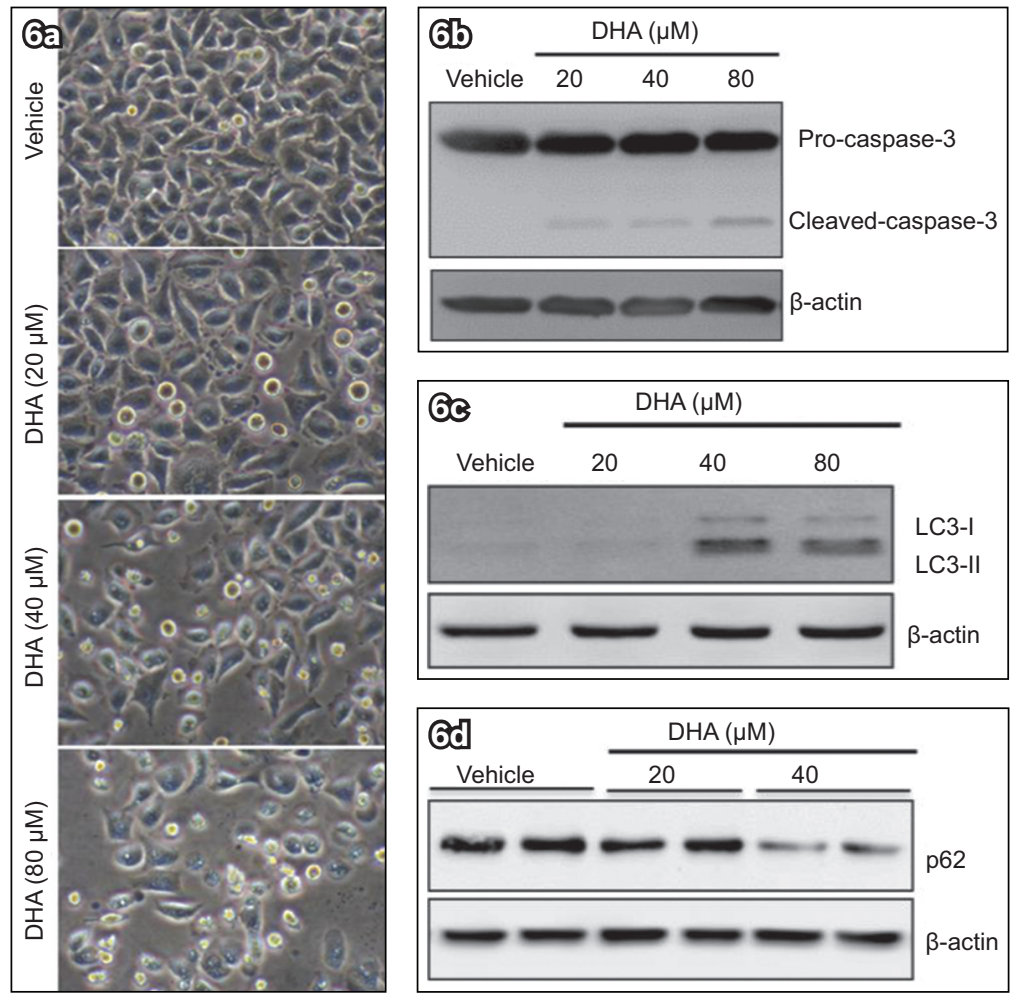

Fig. 6 DHA treatment of HeLa cells induced caspase-3 cleavage and autophagic activation. HeLa cells were treated with different concentrations of DHA for 48 hours. (a) Phase-contrast microscopy image shows elevated and dose-dependent cell death after addition of DHA (immumohistochemical staining, $\times 400$ ). Western blot analyses of (b) caspase-3, (c) LC3 and (d) p62, with $\beta$-actin protein as load control, show that DHA triggered cell death. DHA: dihydroartemisinin; LC3: microtubule-associated protein 1 light chain 3

The cellular response to DHA treatment may include various cell death pathways (e.g. apoptosis, autophagy and necrosis). ${ }^{(21)}$ Caspase-3 is ubiquitously distributed and is a main effector caspase for the apoptotic cascade within cells. ${ }^{(18)}$ We found that cleaved caspase-3 protein levels in cells treated with DHA alone or in combination with cisplatin were significantly higher than those in control cells, and that cleaved caspase- 3 increased significantly in a time-dependent manner in Ishikawa cells, indicating an induction of apoptosis with DHA treatment. DHA treatment resulted in increased LC3-II/LC3-I ratio, decreased p62 protein level and elevated numbers of MDC-positive autophagosomes, suggesting enhanced autophagic activity with DHA treatment. Autophagy plays a dual role in cell survival. ${ }^{(22)}$ We showed that inhibition of autophagy further enhanced cell death, suggesting a cytoprotective function for DHA-induced activation of autophagy. By blocking the autophagy pathway using 3-MA and reducing DHA-induced cancer death, we concluded that the DHA effect against tumour cells was mediated through the autophagy pathway.

Our findings that DHA, with or without cisplatin, significantly induced cell death in the Ishikawa cell line suggest that DHA could also be a good candidate for the treatment of cisplatinresistant endometrial carcinoma. Similar findings were reported for the effects of DHA on cisplatin-resistant ovarian cancer cells (SKOV3/cisplatin). ${ }^{(16)}$ All our results indicate that cisplatin treatment combined with DHA could enhance cisplatin-induced proliferation inhibition or facilitate the killing of cisplatin-resistant cancer cells.

In summary, the present study provides evidence that DHA has a potent antitumour effect against human endometrial cancer cells and cervical cancer cells, likely by facilitating apoptosis as well as reducing the mRNA expression of TfR. We presumed that DHA might cause cell death through the autophagy pathway, which can be influenced by 3-MA. Future studies and further investigation of a possible clinical application of DHA in the treatment of endometrial carcinoma and cervical cancer are warranted. Our results reveal further clues that explain TfR regulation in autophagy and apoptosis induced by DHA. Reactive oxygen species were suggested to be a target of DHA in cancer cell lines, indicating the need for further investigation.

\section{ACKNOWLEDGEMENTS}

This project was supported by National Innovative Experimental Projects of Sichuan University 2012 (201210610114) and New Seed Research Fund of West China Second University Hospital (KX095). We would like to thank Mr Zengliang Xia and Mr Faqiang Zhang at West China Laboratory of Molecular Genetics, Sichuan University, Chengdu, China, for technical assistance.

\section{REFERENCES}

1. Sharma D, Singh G. Thrombocytosis in gynecological cancers. J Cancer Res Ther 2017; 13:193-7.

2. Muliira RS, Salas AS, O'Brien B. Quality of life among female cancer survivors in Africa: an integrative literature review. Asia Pac J Oncol Nurs 2017; 4:6-17.

3. Ben-Arye E, Lavie O, Samuels N, et al. Safety of herbal medicine use during chemotherapy in patients with ovarian cancer: a "bedside-to-bench" approach. Med Oncol 2017; 34:54.

4. Suh DH, Kim M, Kim HJ, Lee KH, Kim JW. Major clinical research advances in gynecologic cancer in 2015. J Gynecol Oncol 2016; 27:e53.

5. Kristeleit RS, Miller RE, Kohn EC. Gynecologic cancers: emerging novel strategies for targeting DNA repair deficiency. Am Soc Clin Oncol Educ Book 2016; 35:e259-68. 
6. Zhang ZS, Wang J, Shen YB, et al. Dihydroartemisinin increases temozolomide efficacy in glioma cells by inducing autophagy. Oncol Lett 2015; 10:379-83.

7. Chen SS, Hu W, Wang Z, Lou XE, Zhou HJ. p8 attenuates the apoptosis induced by dihydroartemisinin in cancer cells through promoting autophagy. Cancer Biol Ther 2015; 16:770-9.

8. Kim SH, Kang SH, Kang BS. Therapeutic effects of dihydroartemisinin and transferrin against glioblastoma. Nutr Res Pract 2016; 10:393-7.

9. Liu L, Wei Y, Zhai S, Chen Q, Xing D. Dihydroartemisinin and transferrin dualdressed nano-graphene oxide for a $\mathrm{pH}$-triggered chemotherapy. Biomaterials 2015; 62:35-46

10. Gutman J, Kovacs S, Dorsey G, Stergachis A, Ter Kuile FO. Safety, tolerability, and efficacy of repeated doses of dihydroartemisinin-piperaquine for prevention and treatment of malaria: a systematic review and meta-analysis. Lancet Infect Dis 2017; 17:184-93.

11. Gaur R, Pathania AS, Malik FA, Bhakuni RS, Verma RK. Synthesis of a series of novel dihydroartemisinin monomers and dimers containing chalcone as a linker and their anticancer activity. Eur J Med Chem 2016; 122:232-46.

12. Que Z, Wang P, Hu Y, et al. Dihydroartemisin inhibits glioma invasiveness via a ROS to P53 to $\beta$-catenin signaling. Pharmacol Res 2017; 119:72-88.

13. Zhang Z, Guo M, Zhao S, Shao J, Zheng S. ROS-JNK1/2-dependent activation of autophagy is required for the induction of anti-inflammatory effect of dihydroartemisinin in liver fibrosis. Free Radic Biol Med 2016; 101:272-83.

14. Tong $\mathrm{Y}$, Liu $\mathrm{Y}$, Zheng $\mathrm{H}$, et al. Artemisinin and its derivatives can significantly inhibit lung tumorigenesis and tumor metastasis through $\mathrm{Wnt} / \beta$-catenin signaling. Oncotarget 2016; 7:31413-28.
15. Omwoyo WN, Melariri P, Gathirwa JW, et al. Development, characterization and antimalarial efficacy of dihydroartemisinin loaded solid lipid nanoparticles. Nanomedicine 2016; 12:801-9.

16. Feng X, Li L, Jiang H, et al. Dihydroartemisinin potentiates the anticancer effect of cisplatin via mTOR inhibition in cisplatin-resistant ovarian cancer cells: involvement of apoptosis and autophagy. Biochem Biophys Res Commun 2014; 444:376-81.

17. Zhai Z, Zhang F, Chen X, et al. Uptake of silver nanoparticles by DHAtreated cancer cells examined by surface-enhanced Raman spectroscopy in a microfluidic chip. Lab Chip 2017; 17:1306-13.

18. Ontikatze T, Rudner J, Handrick R, Belka C, Jendrossek V. Dihydroartemisinin is a hypoxia-active anti-cancer drug in colorectal carcinoma cells. Front Oncol 2014; 4:116.

19. Zhang B, Liu L, Guan H, et al. HepG2 cell cycle related gene transcriptional profiles are altered by a novel vanillin derivative BVAN08. J Med Discov 2017; 2:jmd17036.

20. Chen T, Li M, Zhang R, Wang H. Dihydroartemisinin induces apoptosis and sensitizes human ovarian cancer cells to carboplatin therapy. J Cell Mol Med 2009;13:1358-70.

21. Johansson I, Monsen VT, Pettersen K, et al. The marine n-3 PUFA DHA evokes cytoprotection against oxidative stress and protein misfolding by inducing autophagy and NFE2L2 in human retinal pigment epithelial cells. Autophagy 2015; 11:1636-51.

22. Yao Q, Fu T, Wang LU, et al. Role of autophagy in the $\omega-3$ long chain polyunsaturated fatty acid-induced death of lung cancer A549 cells. Oncol Lett 2015; 9:2736-42 